\title{
PREDICCIÓN FENOLÓGICA DEL CULTIVO DE PAPA MEDIANTE TIEMPO TÉRMICO
}

\author{
PHENOLOGICAL PREDICTION OF POTATO CROP BY MEANS OF THERMAL TIME
}

\author{
Héctor Flores-Magdaleno ${ }^{1}$, Hilario Flores-Gallardo ${ }^{2 \star}$ y Waldo Ojeda-Bustamante ${ }^{3}$
}

\begin{abstract}
${ }^{1}$ Programa de Hidrociencias, Colegio de Postgraduados, Campus Montecillo. Km. 36.5 Carr. México-Texcoco. 56230, Montecillo, Texcoco, Edo. de México, México. ${ }^{2}$ Campo Experimental Valle del Guadiana, Instituto Nacional de Investigaciones Forestales Agrícolas y Pecuarias (INIFAP-CIRNOC). Km. 4.5 Carr. Durango-El Mezquital. 34170, Durango, México. ${ }^{3}$ Instituto Mexicano de Tecnología del Agua. Paseo Cuauhnáhuac 8535, Colonia Progreso. 62550, Jiutepec, Morelos, México.

${ }^{*}$ Autor para correspondencia (flores.hilario@inifap.gob.mx)
\end{abstract}

\section{RESUMEN}

El objetivo del presente estudio fue evaluar tres métodos para predecir la fenología en el cultivo de papa (Solanum tuberosum L.) mediante tiempo térmico, para lo cual se trabajó con un total de 15 parcelas a nivel comercial sembradas con la variedad 'Alpha' en el norte de Sinaloa, México. Los métodos comparados fueron: temperatura media, grados día $\left({ }^{\circ} D\right)$ y días fenológicos de papa $(P$-days $)$, los tres calculados con los datos obtenidos mediante el monitoreo en campo de cada fase fenológica durante los ciclos agrícolas otoño-invierno 2005-2006 y 2006-2007. Los resultados indicaron que el mejor método para predecir la fenología de esta variedad de papa con base en el tiempo térmico fue el $\boldsymbol{P}$-days, porque presentó los menores valores de coeficiente de variación con 0.07 y de desviación estándar con 18.03 para todas las etapas fenológicas analizadas. Al comparar los errores encontrados para cada método en el grupo de parcelas en las que se determinaron las necesidades térmicas más un grupo de otras parcelas usadas para validar dichas necesidades, se confirmó que el método $P$-days tuvo menores errores, pues los valores encontrados fueron 3.6 y $3.2 \%$ para la raíz cuadrada del cuadrado medio del error RMSE y el error medio absoluto $M A E$, respectivamente.

Palabras clave: Solanum tuberosum, grados día, variabilidad climática.

\section{SUMMARY}

The objective of this research was to evaluate three methods to predict potato crop (Solanum tuberosum L.) phenology by means of thermal time, which were applied on 15 commercial plots sowed with the variety 'Alpha' in northern Sinaloa, México. The compared methods were: average temperature, degree days $\left({ }^{\circ} D\right)$ and potato days $(P$-days $)$, all of them calculated with data obtained by field monitoring of each phenological stage during two crop seasons, Autumn-Winter seasons 2005-2006 and 2006-2007. Results showed that the best method to predict the phenology of this potato variety by means of thermal time was the $P$-days technique because it produced the lowest values of variation coefficient with 0.07 and of standard deviation with 18.03, for all the analyzed phenology stages. To compare the errors in thermal requirements obtained with each method in the plots, other plots were used for validation, and so it was confirmed that the P-days method showed the smallest errors, with 3.6 and $3.2 \%$ for the root mean square error RMSE and the mean absolute error MAE, respectively.

Index words: Solanum tuberosum, degree days, climate variability.

\section{INTRODUCCIÓN}

Las estadísticas de la Organización de las Naciones Unidas para la Alimentación y la Agricultura (FAO), indican que a nivel mundial se sembraron casi 17.8 millones de hectáreas del cultivo de papa (Solanum tuberosum L.), con una producción cercana a 352.4 millones de toneladas y un rendimiento promedio de $19.81 \mathrm{t} \mathrm{ha}^{-1}$ en el año 2011 (FAOSTAT, 2013). De acuerdo con Alonso (2002), la papa es una de las principales fuentes de alimentación a nivel mundial después del arroz (Oryza sativa L.), maíz (Zea mays L.) y trigo (Triticum aestivum). En México se sembraron 69,054 ha de papa con un rendimiento promedio de 26.3 $t$ ha $^{-1}$ durante el año agrícola 2011 (SIAP, 2012). En Sinaloa la superficie sembrada de papa, durante el ciclo agrícola otoño-invierno (O-I) 2010-2011, fue de 13,194 ha pero se cosecharon solamente 11,243 ha como consecuencia de las heladas (CONAGUA, 2012).

Desde 1940 en México se cultiva la papa bajo condiciones de temporal (secano) en las sierras y valles altos (principalmente en el área del eje neovolcánico), aunque en las últimas décadas ya se cultiva intensivamente en 23 estados que incluyen la modalidad de riego (Cepeda y Gallegos, 2003). La papa tiene un contenido mayor de nutrientes que los cereales, y es adaptable en los climas frescos ya que no soporta las heladas y los climas cálidos (SEP, 1982). En los últimos años el manejo convencional del cultivo de papa se ha hecho menos funcional debido a la alta variabilidad climática que predomina en las zonas productoras del noroeste de México, lo cual ha generado que los productores apliquen dosis excesivas de insumos que ocasionan contaminación y baja rentabilidad del cultivo (Sifuentes et al., 2013).

El producto final de un cultivo es el resultado de un proceso de actividades agrícolas efectuadas durante todo su ciclo de desarrollo, por lo cual es importante conocer la 
fenología del cultivo, la duración posible de las diferentes fases o etapas, así como sus problemas potenciales y sus necesidades fundamentales (Solórzano, 2007). Los cambios en la morfología externa de la planta, que se expresan con la aparición, transformación o desaparición relativamente rápida de determinados órganos, definen las fases fenológicas o etapas de desarrollo del cultivo, y por tanto la información histórica de la fenología puede ser utilizada en estudios sobre cambio climático global (Elias y Castellvi, 2001). Los actuales cambios en los patrones climáticos demandan el uso frecuente de herramientas para conocer con mayor certidumbre la respuesta de los cultivos en condiciones climáticas contrastantes (Flores-Gallardo et al., 2013).

Para desarrollar una fenología experimental se requiere definir tres factores: el cultivar, las características del suelo, y las condiciones ambientales. El cultivo de papa, en especial, tiene varias fases fenológicas que pueden llegar a ser críticas durante su desarrollo, las cuales son la formación de estolones, la tuberización o formación de tubérculos y la floración. Para elaborar un modelo para la papa, es importante conocer su fenología (Jefferies y Lawson, 1991). Summerfield et al. (1991) proponen que el modelado de la fenología de los cultivos debe ser simple, confiable y plausible desde el punto de vista biológico; según Arazi et al. (1993), estos modelos generalmente se basan en las unidades calor para predecir las fases fenológicas de la planta.

Se han utilizado modelos para predecir la fenología y madurez del melocotón (Prunus persica L. Batsch) mediante variables meteorológicas (Schwartz et al., 1997), y también acoplado con métodos para determinar los grados día $\left({ }^{\circ} \mathrm{D}\right)$ o unidades calor, que permitan predecir la primera y última cosecha del brócoli (Brassica oleracea L. var. 'Itálica') durante varias fechas de siembra (Dufault, 1997). En papa existen modelos de simulación biológica que determinan los días después de la siembra $(d d s),{ }^{\circ} D$, las fases fenológicas y el rendimiento, tales como los modelos: DSSAT (Hoogenboom et al., 2012; Jones et al., 2003), CropSyst (Alva et al., 2010), SPUDSIM (Fleisher et al., 2010), AquaCrop (Raes et al., 2009) y SIMPOTATO (Hodges et al., 1992).

Allen y O'Brien (1986) consideran que los ${ }^{\circ} D$ son un buen indicador para conocer el desarrollo de las fases fenológicas de la papa, pero que los ${ }^{\circ} \mathrm{D}$ no tienen relación con la edad fisiológica de los tubérculos destinados para semilla. Por tanto, el conocimiento de las fases fenológicas del cultivo es importante para acoplar las labores agronómicas y la aplicación de insumos durante su ciclo de desarrollo, ya que el patrón de crecimiento y desarrollo fenológico de otras especies cultivadas como la mandioca (Manihot esculenta Crantz) basado en la acumulación de grados día $\left(\Sigma^{\circ} D\right)$, puede utilizarse para caracterizar el progreso del cultivo en el ambiente del norte de Corrien- tes, Argentina (Burgos et al., 2013).

De acuerdo con Valdez-Torres et al. (2012), el uso de los modelos de predicción fenológica en maíz (Zea mays L.) se utiliza para predecir la aparición de plagas que pueden dañar al cultivo y tomar las medidas pertinentes para su control. En zonas áridas, el efecto de la evaporación en el balance de energía puede afectar fuertemente el registro de la temperatura del aire y en consecuencia el cálculo de los ${ }^{\circ} D$ (Snyder et al., 2001). En México existe poco conocimiento científico generado a nivel local referente a la fenología del cultivo de papa; por lo cual, en la presente investigación se trabajó con parcelas comerciales de papa de la variedad 'Alpha', establecidas en el norte de Sinaloa durante dos ciclos agrícolas otoño-invierno (O-I) 2005-2006 y 2006-2007, para determinar y monitorear las etapas fenológicas en campo con respecto a los $d d s$, lo cual permitió comparar tres métodos para validar la precisión de cada uno en la predicción de la fenología con base en el tiempo térmico, y así determinar el método más viable para la predicción de la fenología en esta variedad de papa cultivada en el Estado de Sinaloa.

\section{MATERIALES Y MÉTODOS}

\section{Localización del área de estudio}

La investigación se hizo con los datos experimentales de Flores-Gallardo et al. (2012), los cuales fueron obtenidos durante dos ciclos agrícolas (O-I) 2005-2006 y 2006-2007 en el distrito de riego 075 "Río Fuerte" (DR-075), que se localiza en las coordenadas geográficas de $25^{\circ} 48.89^{\prime} \mathrm{N}, 109^{\circ}$ $1.53^{\prime} \mathrm{O}$, a una altitud promedio de $20 \mathrm{~m}$, en los módulos de riego Santa Rosa y Taxtes (Cuadro 1). Se utilizaron 15 parcelas de productores cooperantes con una superficie total de 390.84 ha, y la información meteorológica se obtuvo de la red de estaciones agrometeorológicas del DR-075 (Figura $1)$.

\section{Método de la temperatura media o unidades calor}

Para conocer el requerimiento térmico del cultivo con relación a su desarrollo fenológico, el principio de este método es considerar cuando la temperatura media diaria excede un determinado umbral conocido como temperatura base $\left(T_{b}\right)$ (Wang, 1960; Cross y Zuber, 1972; Snyder, 1985). Mediante el método de la temperatura media los ${ }^{\circ} D$ se estiman con la Ecuación 1:

$$
{ }^{\circ} D=\frac{T_{\text {min }}+T_{\max }}{2}-T_{b} \quad \text { Ec. } 1
$$

donde $T_{\min }$ y $T_{\max }$ son las temperaturas mínima y máxima diarias del aire, respectivamente, y $T_{b}$ es la temperatura base o umbral inferior para el inicio del desarrollo del cultivo. 
Cuadro 1. Parcelas comerciales de papa de la variedad 'Alpha' utilizadas para este estudio, que fueron sembradas por Flores-Gallardo et al. (2012).

\begin{tabular}{|c|c|c|c|c|c|}
\hline No. & Parcela & Superficie (ha) & Sistema de riego & Fecha de siembra & Módulo de riego \\
\hline 1 & Las Olivas & 118.32 & Aspersión & Dic-03-2005 & SR \\
\hline 2 & El Campo & 20.00 & Gravedad & Dic-21-2005 & TXT \\
\hline 3 & Zapotillo & 104.00 & Aspersión & Nov-21-2005 & TXT \\
\hline 4 & Cave & 10.00 & Gravedad & Oct-03-2005 & SR \\
\hline 5 & Chispas & 15.00 & Gravedad & Dic-22-2005 & TXT \\
\hline 6 & Guamuchilito & 16.00 & Gravedad & Dic-05-2005 & TXT \\
\hline 7 & Ejido Plan de Ayala 1 & 9.00 & Gravedad & Oct-10-2005 & SR \\
\hline 8 & Ejido Plan de Ayala 2 & 5.35 & Gravedad & Oct-06-2005 & SR \\
\hline 9 & Ejido Plan de Ayala 3 & 9.50 & Gravedad & Oct-07-2005 & SR \\
\hline 10 & Ejido 9 de Diciembre 1 & 9.80 & Gravedad & Oct-05-2005 & SR \\
\hline 11 & Ejido 9 de Diciembre 2 & 9.80 & Gravedad & Oct-11-2005 & SR \\
\hline 12 & Ejido Flores Magón 1 & 18.09 & Gravedad & Oct-14-2005 & SR \\
\hline 13 & Ejido Flores Magón 2 & 18.09 & Gravedad & Nov-17-2005 & SR \\
\hline 14 & Ejido 9 de Diciembre $2^{\dagger}$ & 9.80 & Gravedad & Nov-06-2006 & SR \\
\hline \multirow[t]{2}{*}{15} & Ejido Flores Magón $2^{\dagger}$ & 18.09 & Gravedad & Nov-10-2006 & SR \\
\hline & Total & 390.84 & & & \\
\hline
\end{tabular}

SR: módulo Santa Rosa; TXT: módulo Taxtes; ${ }^{\dagger}$ : ciclo agrícola diferente.

\section{Grados día $\left({ }^{\circ} D\right)$}

Para estimar diariamente los ${ }^{\circ} \mathrm{D}$ con este método, se requiere de la temperatura media ambiental $\left(T_{a}\right)$ y aplicar la Ecuación 2 (Ojeda-Bustamante et al., 2004):

$$
\begin{aligned}
& { }^{\circ} D=T_{a}-T_{c-\text { min }}, T_{a}<T_{c-\text { max }} \\
& { }^{\circ} D=T_{c-m a x}-T_{c-\text { min }} T_{a} \geq T_{c-\max } \\
& { }^{\circ} D=0, T_{a} \leq T_{c-\text { min }}
\end{aligned}
$$

en donde $T_{c-\min }$ y $T_{c-\text { max }}$ son las temperaturas mínima y máxima diarias del aire, respectivamente, y dentro de las cuales se desarrolla la planta en un intervalo de 2 a $29^{\circ} \mathrm{C}$ (FloresGallardo et al., 2012).

\section{Potato days (P-days o días fenológicos de papa)}

De acuerdo con Sands et al. (1979), el cultivo de papa tiene requerimientos térmicos específicos que han sido estudiados con precisión y han conducido al desarrollo de los $P$-days. Este método (Ecuación 3) considera una temperatura mínima de $7^{\circ} \mathrm{C}$ para el desarrollo, una óptima $\left(T_{\text {opp }}\right)$ de $21^{\circ} \mathrm{C}$, y que el desarrollo se detiene a temperaturas mayores o extremas $\left(T_{\text {ext }}\right)$ de $30^{\circ} \mathrm{C}$. Con la información indicada, el método estima los $P$-days de la siguiente manera:

$$
\begin{aligned}
P \text {-days }= & \frac{1}{24} 5 P\left(T_{\min }\right)+8 P\left(\frac{2 T_{\min }+T_{\max }}{3}\right) \\
& +8 P\left(\frac{T_{\text {min }}+2 T_{\text {max }}}{3}\right)+3 P\left(T_{\text {max }}\right)
\end{aligned}
$$

en donde $T_{\max }$ y $T_{\min }$ son las temperaturas máxima y mínima diarias, respectivamente. La función $P(T)$ se calcula con las Ecuaciones 4, 5 y 6, todas con base en el valor de $T$ :

$$
\begin{array}{ll}
P=0, \text { si } T<7 \text { o } T \geq 30 & \text { Ec. } 4 \\
P=k\left[1-\frac{(T-21)^{2}}{(21-7)^{2}}\right], \text { si } \leq T<21 & \text { Ec. } 5 \\
P=k\left[1-\frac{(T-21)^{2}}{(30-21)^{2}}\right], \text { si } 21 \leq T<30 & \text { Ec. } 6
\end{array}
$$

El método asume las siguientes temperaturas para el cultivo de papa: $T_{b}=7{ }^{\circ} \mathrm{C}, T_{\text {opt }}=21^{\circ} \mathrm{C}, T_{\text {ext }}=30^{\circ} \mathrm{C}, \mathrm{y} k$ es un factor de escala con un valor de 10 .

\section{Análisis de datos}

Una vez obtenido el monitoreo en campo de todas las fases fenológicas de la variedad 'Alpha' de papa, el cual consistió en observar el desarrollo de las plantas y registrar la fecha 
en que apareció en campo cada fase de las descritas por Jefferies y Lawson (1991). Lo anterior se llevó a cabo en las 15 parcelas durante los dos ciclos agrícolas y se estimó el tiempo térmico para cada uno de los métodos evaluados.

Se utilizaron las primeras 10 parcelas indicadas en el Cuadro 1 para estimar el tiempo térmico y el promedio para cada etapa fenológica calculada con los tres métodos. Sus coeficientes de variación $(C V)$ se calcularon con la Ecuación 7 y sus desviaciones estándar $(\sigma)$ con la Ecuación 8. El mejor método para pronosticar las fases fenológicas se eligió como el que mostró la menor dispersión. De acuerdo con Perry et al. (1986), el mejor método para pronosticar las fases fenológicas con base en el tiempo térmico es el que presente el menor $C V$.

$$
\begin{array}{ll}
C V=\frac{\sigma}{|\bar{x}|} & \text { Ec. } 7 \\
\sigma=\sqrt{\frac{1}{N-1} \sum_{i=1}^{N}\left(x_{i}-\bar{x}\right)^{2}} & \text { Ec. } 8
\end{array}
$$

donde $\sigma$ es la desviación estándar, $\bar{x}$ es el valor medio, y $x_{i}$ es cada observación registrada.

Un segundo criterio para determinar el mejor método fue calcular el tiempo térmico requerido para cada fase fenológica con el procedimiento descrito anteriormente, $\mathrm{y}$ validando los resultados con las últimas cinco parcelas (Cuadro 1). Como mejor método para pronosticar las fases fenológicas se eligió al que presentó el menor error. Los estadísticos utilizados para medir los errores fueron la raíz cuadrada del cuadrado medio del error (RMSE) y el error medio absoluto (MAE), ambos descritos por Willmott y Matsuura (2005). A continuación se describen las expresiones utilizadas en el cálculo de los estadísticos:

$$
\begin{array}{ll}
\text { RMSE }=\left[N^{-1} \sum_{i=1}^{N}\left(P_{i}-O_{i}\right)^{2}\right]^{0.5} & \text { Ec. } 9 \\
M A E=\left[N^{-1} \sum_{i=1}^{N} a b s\left(P_{i}-O_{i}\right)\right] & \text { Ec. } 10
\end{array}
$$

donde $N$ es el número de observaciones, $P_{i}$ es el valor estimado para cada fase fenológica de las últimas cinco parcelas (Cuadro 1), $O_{i}$ es el valor observado y el cual fue calculado como valor promedio para cada fase fenológica de las primeras 10 parcelas (Cuadro 1).

Por tanto, los valores de RMSE y MAE fueron calculados tanto en ${ }^{\circ} \mathrm{D}$ como también en por ciento (\%) con respecto al tiempo térmico requerido por cada método hasta alcanzar la última etapa fenológica analizada: R3 (tuberización).

\section{Descripción breve de la fenología del cultivo de papa}

Las fases fenológicas monitoreadas en campo fueron las descritas por Jefferies y Lawson (1991), para relacionar su duración promedio con lo observado en campo y su clave correspondiente, aunque se consideró que la siembra y la cosecha no son fases fenológicas sino que se utilizaron para marcar el inicio y termino del cultivo establecido en campo. Por tanto, las principales fases fenológicas son descritas de acuerdo con sus características para realizar una identificación más clara y precisa (Cuadro 2).

Después de simular el desarrollo fenológico del cultivo con cada método propuesto para cada parcela, se hicieron los análisis estadísticos para validar el método más preciso en cuanto a la predicción de la fenología con base en el tiempo térmico acorde a las condiciones de la región de estudio, mediante el uso de la información agrometeorológica de la red de estaciones del DR-075 (Figura 1).

\section{RESULTADOS Y DISCUSIÓN}

Villordon et al. (2009) utilizaron modelos predictivos en camote o papa dulce (Ipomea batatas) para determinar si la relación entre la cosecha y los grados día $\left({ }^{\circ} D\right)$ pueden beneficiar a los productores e investigadores al asegurar el rendimiento máximo y una calidad alta de los tubérculos. De acuerdo con el monitoreo de la fenología en campo, se estimó la duración y los grados día acumulados $\left(\Sigma^{\circ} D\right)$ con el método ${ }^{\circ} D$ para cada fase fenológica con una fecha de siembra promedio en el cultivo de papa (Figura 2).

Según el monitoreo en campo de las fases fenológicas, el cálculo de $\operatorname{los}^{\circ} \mathrm{D}$ por cada método: temperatura media (Figura 3 ), ${ }^{\circ} D$ (Figura 4 ) y $P$-days (Figura 5) en cada parcela, muestran que los métodos tuvieron diferentes tendencias de acumulación térmica atribuibles a la diferencia de escalas que utiliza cada método (Cuadro 3).

Con los tres métodos utilizados para predecir la fenología del cultivo de la papa 'Alpha' con base en el tiempo térmico, el método que presentó menor dispersión para todas las etapas fenológicas analizadas, fue el $P$-days (Cuadro 3) $\mathrm{cu}$ yos valores de $C V$ y de $\sigma$ fueron menores que en los otros dos métodos, y que para todas las etapas fenológicas fueron $C V=0.07$ y $\sigma=18.03$. El uso del $C V$ para seleccionar el mejor método para la predicción de la fenología de la papa está acorde con lo recomendado por Perry et al. (1986), quien lo utilizó para elegir al mejor método de un conjunto de 14 métodos analizados para pronosticar la cosecha de pepino (Cucumis sativus L.). 
Cuadro 2. Principales fases fenológicas del cultivo de papa (adaptado de Jefferies y Lawson, 1991) monitoreadas en campo.

\begin{tabular}{lclc}
\hline No. & Clave & \multicolumn{1}{c}{ Fase fenológica (Descripción) } & Duración promedio (días) \\
\hline 1 & N/A & Siembra & 0 \\
2 & V1 & Crecimiento de yemas y raíces & $0-22$ \\
3 & V2 & Emergencia & $23-32$ \\
4 & V3 & Desarrollo vegetativo e inicio de formación de tubérculos & $33-41$ \\
5 & R1 & Desarrollo de tubérculos (inicio de tuberización) & $42-49$ \\
6 & R2 & Madurez fisiológica de la planta y tubérculos & $50-66$ \\
7 & R3 & Tuberización de tubérculos & $67-115$ \\
8 & N/A & Cosecha & \\
\hline
\end{tabular}

N/A: no aplica.

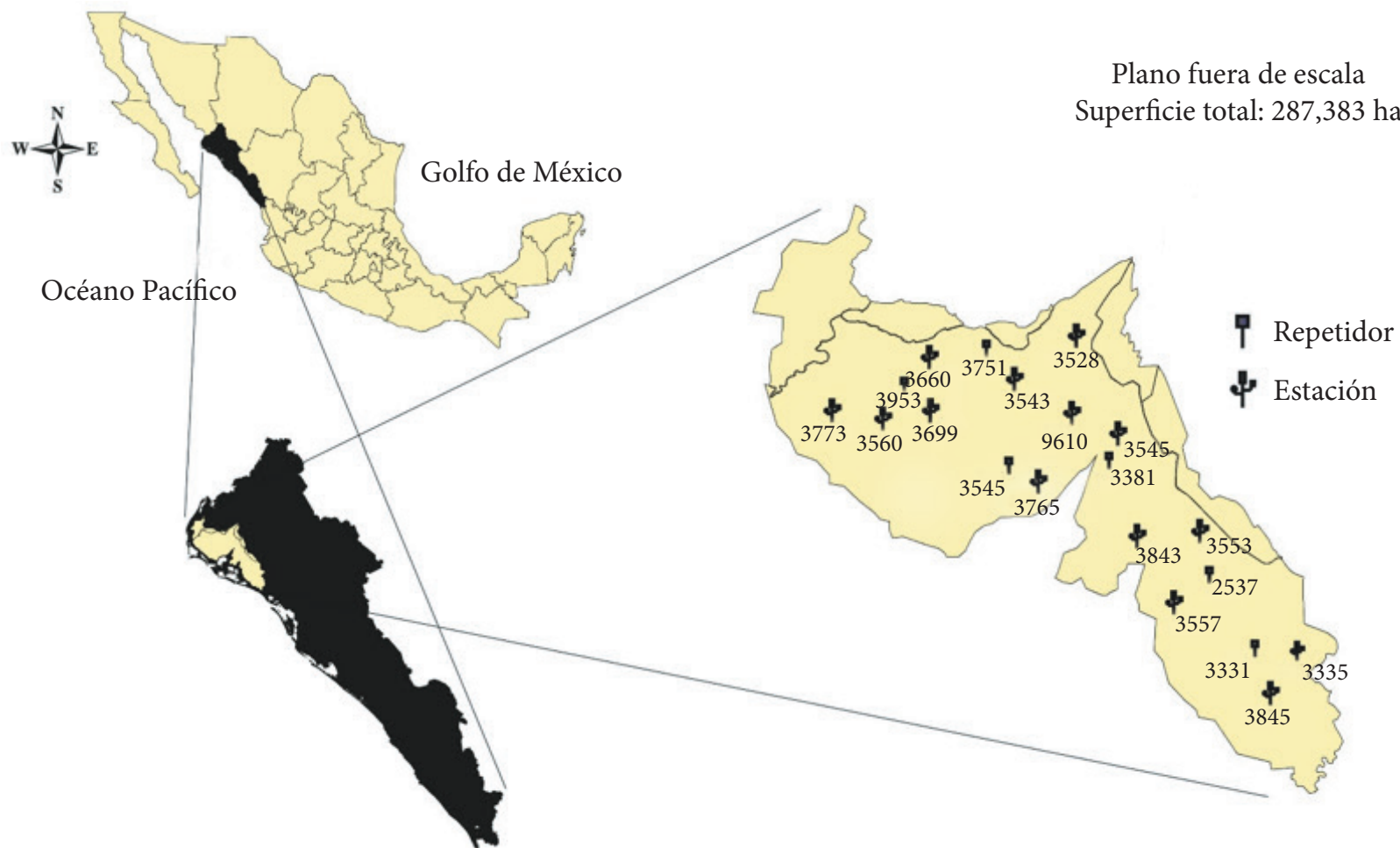

Figura 1. Red de estaciones agrometeorológicas del Distrito de Riego (DR-075) "Río Fuerte" en Sinaloa, México.

El tiempo térmico requerido para cada etapa fenológica y cada método utilizado (Cuadro 3), constituye información útil en la planeación de las actividades agrícolas del cultivo de papa. El conocimiento de los requerimientos térmicos para cada etapa fenológica, representa ventajas prácticas y agronómicas, que permiten optimizar la utilización de insumos y planificar el cultivo de papa bajo condiciones climáticas variables.

Los resultados encontrados (Cuadro 4) al considerar como valores reales al tiempo térmico promedio calculado con las primeras 10 parcelas para cada etapa fenológica y cada método utilizado (Cuadro 3), fueron comparados con los valores observados para todas las etapas fenológicas y cada método utilizado para las últimas cinco parcelas (Cuadro 1). Los valores de la raíz cuadrada del cuadrado medio del error (RMSE) y el error medio absoluto (MAE) fueron menores para el método de $P$-days cuando fueron expresados tanto en ${ }^{\circ} D$ como en $\%$, con respecto al tiempo térmico requerido para alcanzar la etapa de tuberización (R3). Este resultado concuerda con el análisis del coeficiente de variación $(C V)$ en el que también el método $P$-days fue el que presentó menor dispersión de datos.

De acuerdo con Cantos-de-Ruiz et al. (1989), las altas y bajas temperaturas influyen en gran medida en la pérdida 


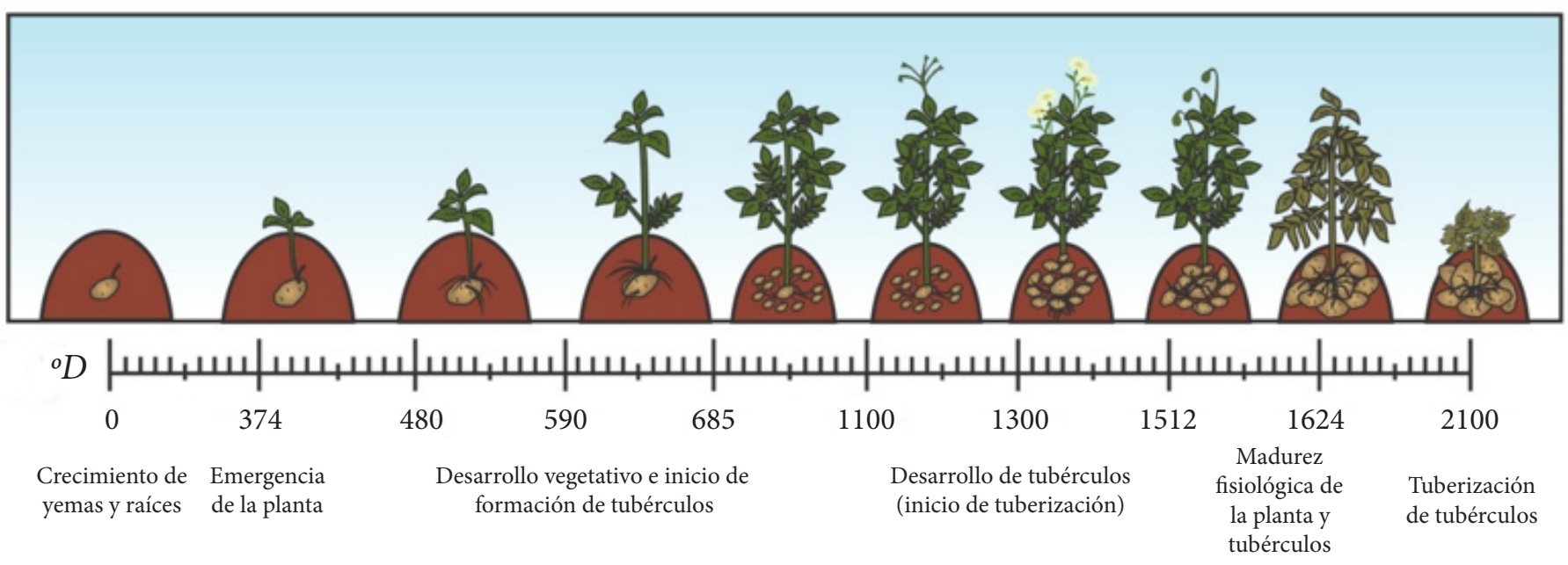

Figura 2. Estimación de la duración y acumulación de grados día $\left(\Sigma^{\circ} D\right)$ de las fases fenológicas para una fecha de siembra promedio, de acuerdo con el monitoreo en campo y con el método ${ }^{\circ} \mathrm{D}$ (elaboración propia a partir de la información de Jefferies y Lawson, 1991).

(a)

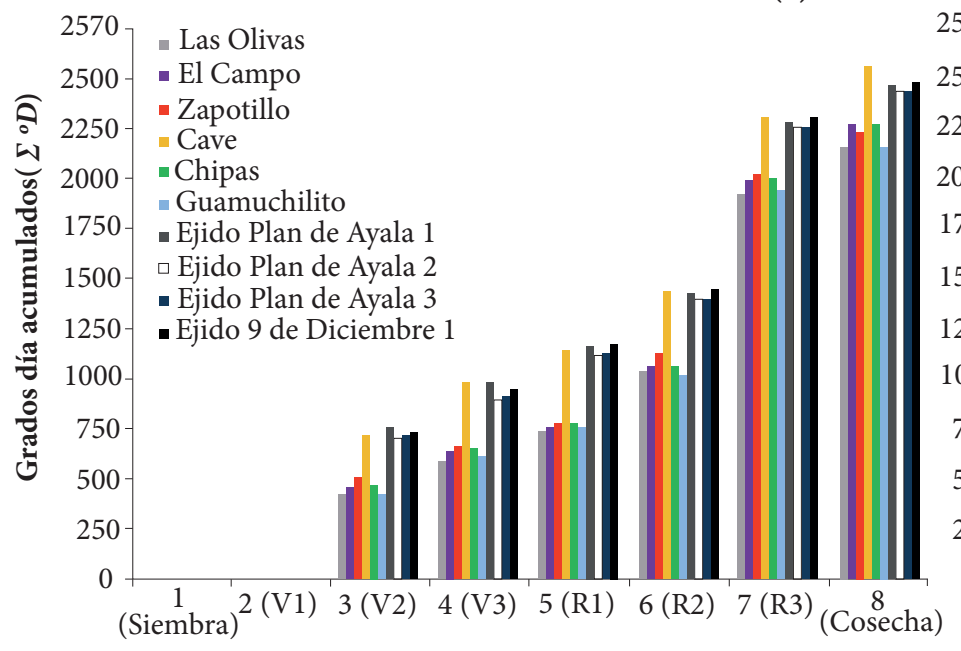

Fase fenológica a partir de la siembra hasta la cosecha

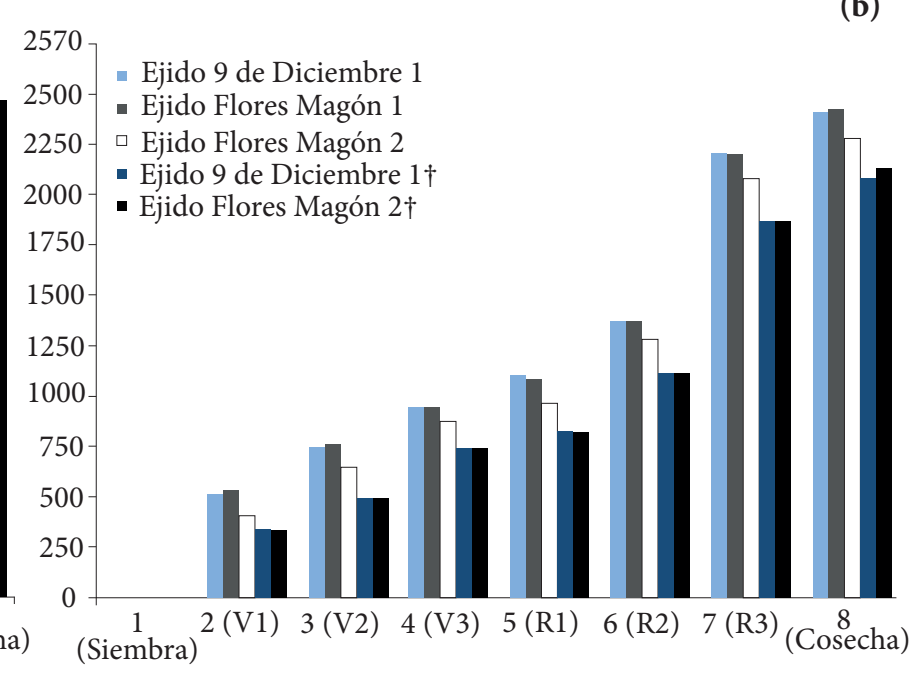

Fase fenológica a partir de la siembra hasta la cosecha

Figura 3. Acumulación de grados día $\left(\Sigma^{\circ} D\right)$ calculados con el método de la temperatura media, para las primeras 10 parcelas analizadas (a) y las cinco parcelas de validación (b).

respiratoria y en el desarrollo del follaje inicial del cultivo. Por tanto, la predicción fenológica con base en el tiempo térmico es una alternativa para predecir las diferentes etapas fenológicas del cultivo de papa con respecto a las condiciones ambientales de la zona de estudio, lo que permite determinar la aplicación de insumos agrícolas así como la implementación oportuna de labores culturales.

\section{CONCLUSIONES}

De los tres métodos analizados, el método $P$-days fue el más preciso en la predicción de las fases fenológicas en el cultivo de la variedad 'Alpha' de papa con base en el tiempo térmico, ya que presentó los valores más pequeños de dispersión, cuyos valores promedio para todas las etapas fenológicas analizadas fueron de 0.07 y de 18.03 para el $C V$ $\mathrm{y} \sigma$, respectivamente.

Al comparar los errores encontrados con cada método en un grupo de parcelas para determinar las necesidades térmicas y otro grupo de parcelas para validar dichas necesidades, se encontró una vez más que el método $P$-days fue el que presentó menores errores, con valores de 3.6 y 3.2 $\%$ para la raíz cuadrada del cuadrado medio del error y el 
(a)

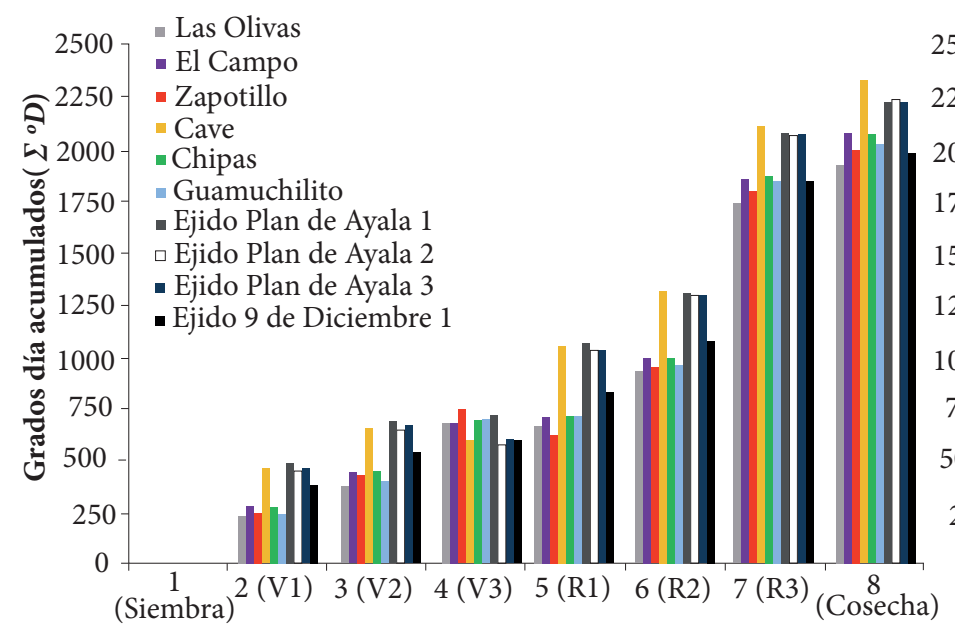

Fase fenológica a apartir de la simbre hasta la cosecha

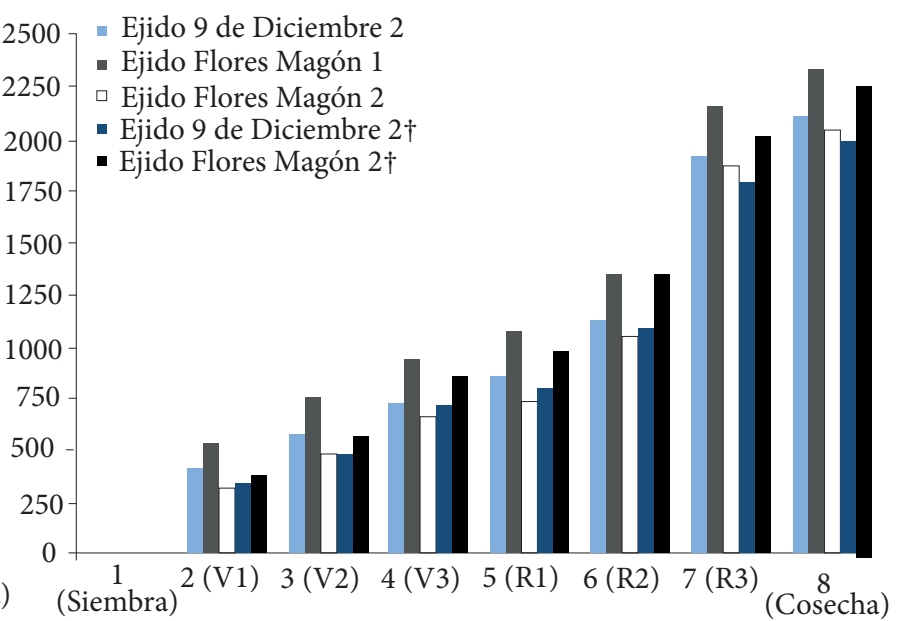

Fase fenológica a apartir de la simbre hasta la cosecha

Figura 4. Acumulación de grados día $\left(\Sigma^{\circ} \mathrm{D}\right)$ calculados con el método ${ }^{\circ} \mathrm{D}$, para las primeras 10 parcelas analizadas $(\mathrm{a})$ y las cinco parcelas de validación $(b)$.

(a)

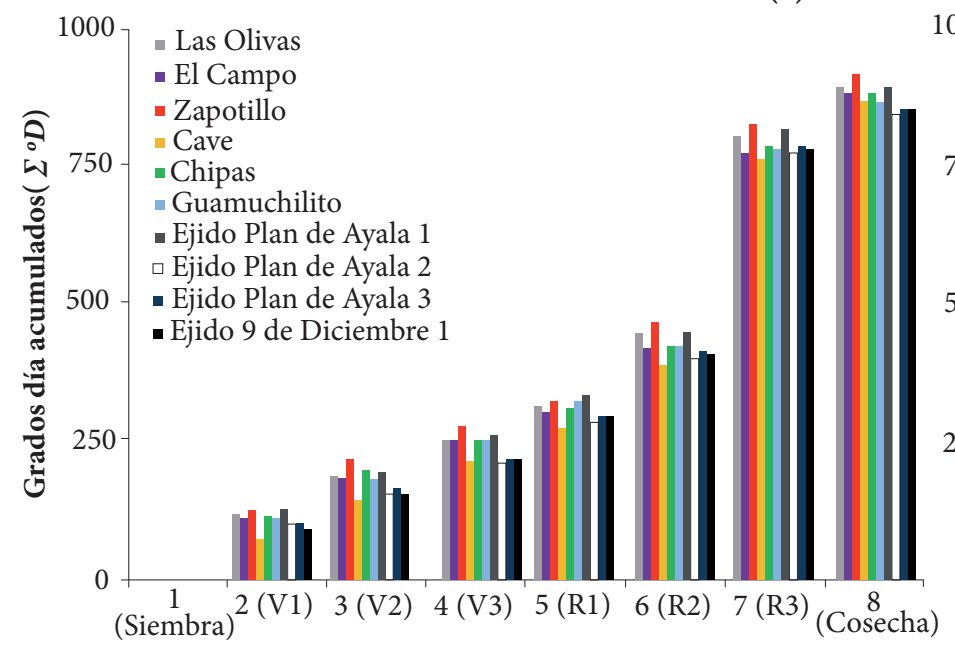

Fase fenológica a apartir de la simbre hasta la cosecha (b)

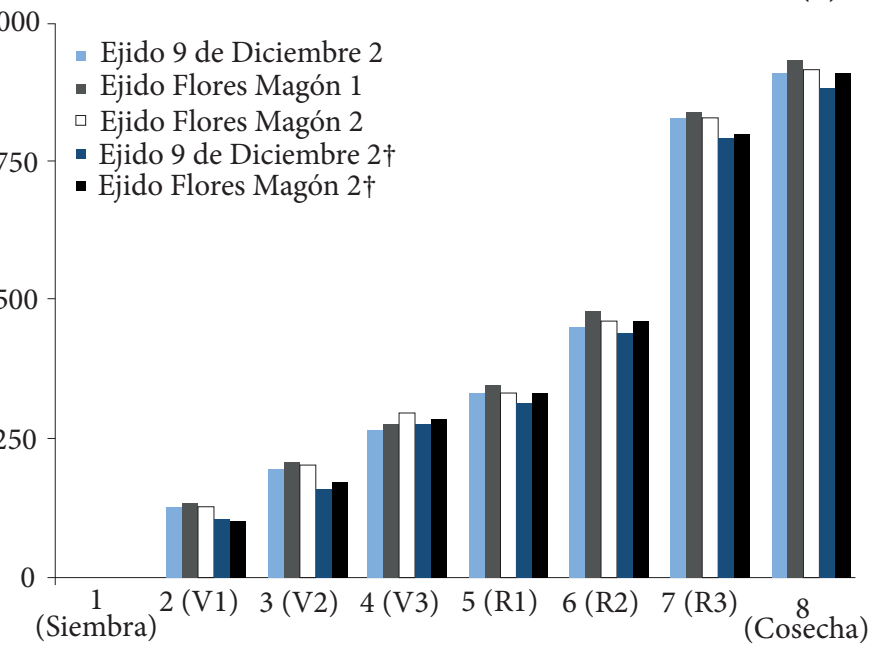

Fase fenológica a apartir de la simbre hasta la cosecha

Figura 5. Acumulación de grados día $\left(\Sigma^{\circ} D\right)$ calculados con el método $P$-days, para las primeras 10 parcelas analizadas $($ a) y las cinco parcelas de validación (b).

error medio absoluto, respectivamente.

\section{BIBLIOGRAFÍA}

Allen E. J. and P. J. O'Brien (1986) The practical significance of accumulated day-degrees as a measure of physiological age of seed potato tubers. Field Crops Research 14:141-151.

Alonso A. F. (2002) El Cultivo de la Patata. $2^{\mathrm{a}}$ ed. Ed. Mundi-Prensa. Madrid, España. $381 \mathrm{p}$.

Alva A. K., J. Marcos, C. Stockle, V. R. Reddy and D. J. Timlin (2010) A crop simulation model for predicting yield and fate of nitrogen in irrigated potato rotation cropping system. Journal of Crop Improvement 24:142-152.

Arazi Y. and S. Wolf, A. Marani (1993) A prediction of developmental stages in potato plants based on the accumulation of heat units. Agricultural Systems 43:35-50.

Burgos A. M., J. Prause, J. A. Argüello y P. J. Cenóz (2013) Fenología de los estados vegetativos de mandioca (Manihot esculenta Crantz) en base al tiempo térmico. Revista de la Facultad de Ciencias Agrarias de la Universidad de Cuyo 45:43-52.

Cantos-de-Ruiz S. T., F. H. Andrade y A. Mendiburu (1989) Rendimiento potencial del cultivo de papa en Balcarce, causas que limitan la productividad real. Revista Latinoamericana de la Papa 2:29-45

Cepeda S. M. y M. G. Gallegos (2003) La Papa "El Fruto de la Tierra". 1a ed. Ed. Trillas. México, D.F. 251 p.

CONAGUA, Comisión Nacional del Agua (2012) Estadísticas Agrícolas de los Distritos de Riego. Año Agrícola 2010-2011. Subdirección General de Infraestructura Hidroagrícola. México, D.F. 339 p.

Cross H. Z. and M. S. Zuber (1972) Prediction of flowering dates in maize 
Cuadro 3. Análisis estadístico para los tres métodos de predicción de la fenología en papa de la variedad 'Alpha'.

\begin{tabular}{|c|c|c|c|c|c|c|}
\hline \multirow[b]{2}{*}{ No. } & \multirow[b]{2}{*}{ Clave } & \multirow[b]{2}{*}{ Fase fenológica } & & \multicolumn{3}{|c|}{ Método } \\
\hline & & & & $\begin{array}{l}\text { Temperatura } \\
\text { media }\end{array}$ & ${ }^{\circ} \mathrm{D}$ & $P$-days \\
\hline \multirow[t]{3}{*}{1} & N/A & Siembra & & N/A & N/A & $\mathrm{N} / \mathrm{A}$ \\
\hline & & & Promedio & 397.22 & 355.60 & 111.46 \\
\hline & & & $C V$ & 0.29 & 0.29 & 0.15 \\
\hline \multirow[t]{3}{*}{2} & V1 & Crecimiento de yemas y raíces & $\sigma$ & 116.41 & 102.58 & 16.40 \\
\hline & & & Promedio & 591.10 & 528.51 & 178.59 \\
\hline & & & $C V$ & 0.24 & 0.24 & 0.11 \\
\hline \multirow[t]{3}{*}{3} & V2 & Emergencia & $\sigma$ & 142.69 & 125.05 & 20.46 \\
\hline & & & Promedio & 787.78 & 705.09 & 244.11 \\
\hline & & & $C V$ & 0.21 & 0.21 & 0.09 \\
\hline \multirow[t]{3}{*}{4} & V3 & $\begin{array}{l}\text { Desarrollo vegetativo e inicio de forma- } \\
\text { ción de tubérculos }\end{array}$ & $\sigma$ & 167.53 & 144.77 & 22.84 \\
\hline & & & Promedio & 951.55 & 845.30 & 306.30 \\
\hline & & & $C V$ & 0.21 & 0.21 & 0.06 \\
\hline \multirow[t]{3}{*}{5} & $\mathrm{R} 1$ & $\begin{array}{l}\text { Desarrollo de tuberculos (1nicio de tuber1- } \\
\text { zación) }\end{array}$ & $\sigma$ & 203.18 & 178.29 & 18.96 \\
\hline & & & Promedio & 1242.78 & 1107.29 & 424.98 \\
\hline & & & $C V$ & 0.16 & 0.15 & 0.06 \\
\hline \multirow[t]{2}{*}{6} & $\mathrm{R} 2$ & $\begin{array}{l}\text { Madurez fisiologica de la planta y tuber- } \\
\text { culos }\end{array}$ & $\sigma$ & 194.45 & 165.40 & 24.36 \\
\hline & & & Promedio & 2135.12 & 1924.98 & 788.48 \\
\hline \multirow{2}{*}{7} & $\mathrm{R} 3$ & Tuberización de tuhérculos & $C V$ & 0.08 & 0.07 & 0.03 \\
\hline & $\mathrm{NJ}$ & IUverizacton de tudercuios & $\sigma$ & 166.70 & 138.94 & 20.01 \\
\hline \multirow{3}{*}{8} & $N / A$ & Cocoche & & N/A & N/A & N/A \\
\hline & $10 / \pi$ & Cosectia & $C V$ & 0.16 & 0.15 & 0.07 \\
\hline & & Promedio todas las etapas & $\sigma$ & 141.90 & 123.16 & 18.03 \\
\hline
\end{tabular}

N/A: no aplica. C. V. = coeficiente de variación; $\sigma=$ desviación estándar.

Cuadro 4. Análisis estadístico realizado entre dos grupos de parcelas, 10 parcelas para estimación de requerimientos térmicos y cinco parcelas para la validación.

\begin{tabular}{|c|c|c|c|c|}
\hline Método & RMSE ( $\left.{ }^{\circ} \mathrm{D}\right)$ & $M A E\left({ }^{\circ} D\right)$ & RMSE $(\%)^{\dagger}$ & MAE $(\%)^{\dagger}$ \\
\hline Temperatura media & 134 & 118 & 6.3 & 5.5 \\
\hline${ }^{\circ} \mathrm{D}$ & 116 & 96 & 6.0 & 5.0 \\
\hline$P$-days & 28 & 25 & 3.6 & 3.2 \\
\hline
\end{tabular}


based on different methods of estimating thermal units. Agronomy Journal 64:351-355.

Dufault R. J. (1997) Determining heat unit requirements for broccoli harvest in coastal South Carolina. Journal of American Society of Horticultural Science 122:169-174.

Elías C. F. y S. F. Castellví (2001) Agrometeorología. 2a ed. Ed. MundiPrensa. Madrid, España. 517 p.

FAOSTAT, Food and Agriculture Organization Statistical (2013) FAO Statistical Database 2012. Food and Agriculture Organization of the United Nations (FAO). Rome, Italy. Available at: http:// faostat3.fao.org/home/index.html

Fleisher D. H., D. J. Timlin, Y. Yang and V. R. Reddy (2010) Simulation of potato gas exchange rates using SPUDSIM. Agricultural and Forest Meteorology 150:432-442.

Flores-Gallardo H., W. Ojeda-Bustamante, H. Flores-Magdaleno, E. Mejía-Sáenz y E. Sifuentes-Ibarra (2012) Grados día y la programación integral del riego en el cultivo de papa. Terra Latinoamericana 30:59-67.

Flores-Gallardo H., W. Ojeda-Bustamante, H. Flores-Magdaleno, E. Sifuentes-Ibarra y E. Mejía-Sáenz (2013) Simulación del rendimiento de maíz (Zea mays L.) en el norte de Sinaloa usando el modelo AquaCrop. Agrociencia 47:347-359.

Hodges T., S. L. Johnson and B. S. Johnson (1992) A modular structure for crop simulation models: implemented in the SIMPOTATO model. Agronomy Journal 84:911-915.

Hoogenboom G., J. W. Jones, P. W. Wilkens, C. H. Porter, K. J. Boote, L. A. Hunt, U. Singh, J. L. Lizaso, J. W. White, O. Uryasev, F. S. Royce, R. Ogoshi, A. J. Gijsman, G. Y. Tsuji and J. Koo (2012) Decision Support System for Agrotechnology Transfer (DSSAT) Version 4.5 [CD-ROM]. University of Hawaii, Honolulu, Hawaii. Available at: http://dssat.net/

Jefferies R. A. and H M Lawson (1991) A key for the stages of development of potato (Solanum tuberosum). Annals of Applied Biology 119:387-399.

Jones J. W., G. Hoogenboom, C. H. Porter, K. J. Boote, W. D. Batchelor, L. A. Hunt, P. W. Wilkens, U. Singh, A. J. Gijsman and J. T. Ritchie (2003) DSSAT cropping system model. European Journal of Agronomy 18:235-265.

Ojeda-Bustamante W., E. Sifuentes-Ibarra, D. C. Slack and M. Carrillo (2004) Generalization of irrigation scheduling parameters using the growing degree days concept: application to a potato crop. Irrigation and Drainage 53:251-261.

Perry K. B., T. C. Wehner and G. L. Johnson (1986) Comparison of 14 methods to determine heat unit requirements for cucumber harvest. HortScience 21:419-423.

Raes D., P. Steduto, T. C. Hsiao and E. Fereres (2009) AquaCrop. Reference Manual. FAO, Rome, Italy. 218 p.
Sands P. J., C. Hackett and H. A. Nix (1979) A model of the development and bulking of potatoes (Solanum tuberosum L.). I. Derivation from well-managed field crops. Field Crops Research 2:309-331.

Schwartz M. D., G. J. Carbone, G. L. Reighard and W. R. Okie (1997) A model to predict peach phenology and maturity using meteorological variables. HortScience 32:213-216.

SEP, Secretaría de Educación Pública (1982) Papas. Manuales para Educación Agropecuaria. Ed. Trillas. México, D.F. 54 p.

SIAP, Servicio de Información Agroalimentaria y Pesquera (2012) Atlas agropecuario y pesquero: información del sector agroalimentario 2012. Servicio de información agroalimentaria y pesquera. Secretaría de Agricultura, Ganadería, Desarrollo Rural, Pesca y Alimentación (SAGARPA). México. 154 p. Available at: http://www.siap.gob.mx

Sifuentes E., W. Ojeda, C. Mendoza, J. Macías, J. del R. Ruelas y M. A. Inzunza (2013) Nutrición del cultivo de papa (Solanum tuberosum L.) considerando variabilidad climática en el "Valle del Fuerte" Sinaloa, México. Revista Mexicana de Ciencias Agrícolas 4:585-597.

Snyder R. L. (1985) Hand calculating degree days. Agricultural and Forest Meteorology 35:353-358.

Snyder R. L., D. Spano, P. Duce and C. Cesaraccio (2001) Temperature data for phenological models. International Journal of Biome teorology 45:178-183.

Solórzano V. E. (2007) Guías Fenológicas para Cultivos Básicos. Ed. Trillas. México, D.F. 152 p.

Summerfield R. J., E. H. Roberts, R. H. Ellis and R. J. Lawn (1991) Towards the reliable prediction of time to flowering in six annual crops. I. The development of simple models for fluctuating field environments. Experimental Agriculture 27:11-31.

Valdez-Torres J. B., F. Soto-Landeros, T. Osuna-Enciso y M. A. BáezSañudo (2012) Modelos de predicción fenológica para maíz blanco (Zea mays L.) y gusano cogollero (Spodoptera frujiperda J. E. Smith). Agrociencia 46:399-410.

Villordon A., C. Clark, D. Ferrin and D. LaBonte (2009) Using growing degree days, agrometeorological variables, linear regression, and data mining methods to help improve prediction of sweetpotato harvest date in Louisiana. HortTechnology 19:133-144.

Wang J. Y. (1960) A critique of the heat unit approach to plant response studies. Ecology 41:785-790.

Willmott C. J. and K. Matsuura (2005) Advantages of the mean absolute error (MAE) over the root mean square error (RMSE) in assessing average model performance. Climate Research 30:79-82. 